\title{
DILEMAS BIOÉTICOS EN TORNO A LA FERTILIZACIÓN IN VITRO (FIV) Y LA MUJER GESTANTE: HACIA LA FIGURA DE UN CONSEJERO REPRODUCTIVO
}

Ana Violeta Trevizo ${ }^{1}$

Resumen: Este artículo plantea algunos dilemas importantes en torno a la fertilización in vitro (FIV) y la mujer gestante. En él se contrastan las dos posturas bioéticas predominantes: liberal y conservadora respecto de ambos fenómenos. Se llevó a cabo un análisis cualitativo respecto a las posturas teóricas dominantes y el estado del arte de las tecnologías reproductivas y la gestación subrogada. En conclusión, se propone una figura bioética innovadora en la práctica de la reproducción asistida: un consejero reproductivo que ayude en la toma de decisiones durante el proceso de reproducción artificial de la pareja o persona sola, y sea un interlocutor entre ellos y el especialista. Además, se proponen mínimos éticos para la selección de la mujer gestante.

Palabras clave: dilemas bioéticos, tecnologías reproductivas, mujer gestante, consejero reproductivo

Bioethical dilemmas around in vitro fertilization (IVF) and gestational woman: towards the figure of a reproductive counselor

Abstract: This paper studies some important dilemmas around in vitro fertilization (IVF) and gestational woman. Two predominant bioethical views are contrasted: liberal and traditional with respect to both phenomena. Qualitative analyses were carried out with respect to dominant theoretical views and the state of the art of reproductive technologies and subrogate pregnancy. As conclusion, an innovative bioethical figure in the practice of assisted reproduction is proposed: a reproductive counselor that helps in decision making during the process of artificial reproduction of the couple or single person to be an interlocutor between the users and the specialist. Furthermore, an ethical minimum for the selection of gestational women is proposed.

Key words: bioethical dilemmas, reproductive technologies, gestational women, reproductive counselor

Dilemas bioéticos em torno da fertilizaçáo in vitro (FIV) e a mulher gestante: em torno da figura de um conselheiro reprodutivo

Resumo: Este artigo propóe alguns dilemas importantes em torno da fertilização in vitro (FIV) e a mulher gestante. Nele se contrastam as duas posturas bioéticas predominantes: liberal e conservadora, a respeito de ambos os fenômenos. Foi levada a cabo uma análise qualitativa a respeito das posturas teóricas dominantes e do estado da arte das tecnologias reprodutivas e da gestação substituta. Em conclusão, se propõe uma figura bioética inovadora na prática da reprodução assistida: um conselheiro reprodutivo que ajude na tomada de decisóes durante o processo de reprodução artificial do casal ou de apenas uma pessoa, e seja um interlocutor entre eles e o especialista. Ademais, se propóem mínimos éticos para a seleção da mulher gestante.

Palavras-chave: dilemas bioéticos, tecnologias reprodutivas, mulher gestante, conselheiro reprodutivo

\footnotetext{
${ }^{1}$ Programa de Maestría y Doctorado en Ciencias Médicas, Odontológicas y de la Salud, Facultad de Medicina. Unidad de Posgrado de la Universidad Nacional Autónoma de México, México

Correspondencia: avtg@hotmail.com
} 


\section{Introducción}

Las nuevas posibilidades reproductivas han dado origen a las ideas de "autonomía de la reproducción" y de "libertad reproductiva", las cuales vienen muy bien en un mundo que abre cada vez más posibilidades tecnológicas. Sin embargo, las tecnologías reproductivas que analizamos en este artículo presentan dilemas bioéticos desde el inicio del tratamiento y antes de implantar los embriones en el útero de la mujer gestante, para lo cual debe producirse un número de embriones razonable. Estos dilemas no solo se relacionan con cuántos embriones producir, sino también con cuántos ciclos de implantación son adecuados para afectar lo menos posible a la mujer gestante. Después, habrá que seleccionar los embriones más viables y, en casos particulares, hacer exámenes genéticos. El destino final de los embriones sobrantes será una decisión dilemática para la pareja, pues puede destruirlos, donarlos o crioconservarlos; en esta última opción debe definir por cuánto tiempo y para qué se desean conservar.

Además, en relación con la tecnología que intervendrá el cuerpo de la mujer surgen otros problemas para ella, sea donante o gestante, como la instrumentalización de su cuerpo, que puede llegar al extremo de generar un mercado o "turismo reproductivo" si las condiciones económicas de su país lo promueven, ya sea en países con economías emergentes, en donde las mujeres padecen pobreza extrema y no existe reglamentación para las tecnologías reproductivas, o en países desarrollados, en los cuales se fomenta esta industria de manera abierta con la legislación correspondiente y la mujer, de manera informada y autónoma, pone a la venta sus células germinales o renta su útero.

También surgen dilemas en relación a los diferentes formatos de pareja y familia. La fertilización in vitro (FIV) y la gestación subrogada sin duda ofrecen la oportunidad a nuevos formatos como los mencionados por Charlesworth $(1)^{2}$. Los dile-

\footnotetext{
${ }^{2}$ Diversos formatos de familia en las sociedades contemporáneas según Charlesworth: los hijos que nacen de parejas heterosexuales en matrimonio, de parejas heterosexuales en uniones de facto, hijos nacidos de matrimonios previos, después del divorcio y nuevo matrimonio, hijos nacidos de padres o madres solteras, hijos adoptados, hijos nacidos de parejas heterosexuales mediante la inseminación artificial de un donante, hijos nacidos de parejas homosexuales a través de la inseminación artificial de un donante, hijos nacidos de gametos
}

mas surgen cuando no está clara la filiación con el nacido respecto de sus padres legales, si la fertilización y la subrogación se realizan fuera del país de origen de los mismos. El registro y la nacionalidad de los nacidos pueden negarse en caso de que la ley en el país de origen de estos prohíba la gestación subrogada o la unión entre parejas del mismo sexo. Este vacío jurídico puede privar a uno de ellos o a ambos de la filiación con el niño y retenerlo en el país de nacimiento, e incluso privarlo de nacionalidad y quedar bajo la tutela de la mujer gestante y no de los padres legales (los cuales gestionaron el nacimiento del bebé). Además de que la pareja o soltero que solicita la gestación pueden ser sometidos a procesos legales largos y desgastantes.

Los infantes nacidos por FIV presentarán quizá dilemas relacionados con su origen genético. Por un lado, tienen derecho a conocerlo y, por otro, se debe respetar la confidencialidad de la información del donante o donantes (en la mayoría de los países no es posible acceder a sus datos personales). También puede haber problemas con la filiación del niño si la mujer gestante dio sus óvulos para la fertilización y desea quedarse con él. Y aunque no haya dado sus óvulos, la gestante puede querer quedarse con el niño. Asimismo, los nacidos por FIV tendrán que enfrentar las expectativas de los padres legales, porque fueron escogidos por ellos, e incluso, en algunos casos, escogieron el sexo de los embriones. En especial, el nacido deberá enfrentar la expectativa de buena salud, si se eliminaron embriones con enfermedades genéticas, lo que no excluye que puedan padecer enfermedades de otra índole.

En este artículo se contrastan la postura liberal y la conservadora ante la FIV y gestación subrogada, con la finalidad de resaltar las ventajas en el discurso de ambas miradas y, de este modo, tratar de encontrar equilibrio entre lo que aporta cada postura respecto de ambos fenómenos y el diálogo entre ellas. De igual forma, se sugieren límites de la autonomía reproductiva ${ }^{3}$, con la finalidad

de una pareja a través de la fertilización in vitro y transferencia embrionaria, hijos nacidos de gametos de donantes o de embriones de donantes a través de la fertilización in vitro y transferencia embrionaria, hijos nacidos del acuerdo de gestación subrogada a través de la fertilización in vitro homóloga o heteróloga, etc.

${ }^{3}$ Para alcanzar una verdadera autonomía reproductiva existen algunas condiciones, como que dicha autonomía debe ser racional, reflexiva y ética, y no una simple manifestación de deseo. 
de proteger a la mujer gestante y a los posibles nacidos por FIV.

También se analizan las responsabilidades bioéticas en la reproducción asistida. Entre ellas: defender la dignidad de las personas vulnerables; cuidar la seguridad de los tratamientos de fertilidad; establecer con claridad los lineamientos para su aplicación; contar con la infraestructura adecuada, no solo para realizar la fertilización sino para el almacenamiento de gametos y embriones; contar con guías clínicas y protocolos para el manejo de las personas en tratamientos de fertilidad; contemplar que a la pareja o persona sola que solicita la FIV se les haga una previa evaluación psicológica, al igual que a la mujer gestante, y dar seguimiento durante toda la gestación.

Por último, se propone la figura de un "consejero reproductivo", con la finalidad de facilitar la toma de decisiones y ser interlocutor entre los que solicitan la FIV y el médico especialista en reproducción. El "consejero reproductivo" ayudaría en el proceso de toma de decisiones de la pareja o persona sola, además, en caso de necesitarlo, puede auxiliarlos a elegir a una mujer gestante que cumpla con algunos mínimos éticos que serán tratados en este artículo. Además, puede ayudar a los solicitantes de la FIV y mujer gestante a comprender la información técnica sobre los procedimientos, las implicaciones éticas en relación con todos los participantes y el tratamiento, así como orientarlos en la toma de decisiones respecto del número de intentos y de embriones a implantar, el uso de células germinales o embriones de donantes, entre otras situaciones que puedan presentarse durante el tratamiento de fertilidad, e incluso después del nacimiento mediante FIV y gestación subrogada.

Posturas bioéticas predominantes ante la FIV y gestación subrogada: liberal y conservadora

El diálogo en bioética es indispensable y, como afirma la bioeticista mexicana Lizbeth Sagols, ello constituye una "interfaz", pues este concepto alude de forma simultánea a la unión y encuentro entre dos fenómenos y a su dinamismo, su diferencia. Se trata pues del diálogo entre los pro y los contra de las innovaciones tecnológicas, aquellas que pueden afectar la condición humana, sin caer en las posturas extremas de tecnofanatismo o tec- $\operatorname{nofobia}(2)$.

La postura conservadora se centra en los riesgos para la mujer gestante y para los nacidos, y en algunas consecuencias sociales (aunque sobre este punto no hay evidencia concluyente). Esta postura argumenta en contra de la posible manipulación genética de los embriones a implantar, la ausencia de confidencialidad que pudiera darse en los tratamientos de reproducción asistida, entre otros problemas que pueden surgir por el uso de tecnologías reproductivas.

León Kass, bioeticista conservador, critica fuertemente las tecnologías de la reproducción. Argumenta que nos hemos acostumbrado a las nuevas prácticas de reproducción humana; no solo a la FIV, sino también a la manipulación, donación de embriones y al alquiler de úteros. Afirma que la bioética se ha contentado con analizar argumentos morales, reaccionando frente a los nuevos desarrollos tecnológicos e incorporando los problemas emergentes de política pública; todo esto realizado con la fe ingenua de que nuestros males pueden evitarse mediante la compasión, la regulación y el respeto por la autonomía. Para él, la procreación humana no es una simple actividad de nuestra voluntad racional; es una actividad más completa, pues nos compromete tanto en forma corporal, como erótica, espiritual y racional. Sostiene que hay sabiduría en el misterio de la naturaleza, que ha unido el placer del sexo, el mudo deseo de la unión, la comunicación del acto de amor y el deseo tan profundo pero parcialmente articulado de tener hijos, en la misma actividad por la cual continuamos la cadena de la existencia y participamos en la renovación de la posibilidad humana. Sabiéndolo o no, separar la procreación del sexo, el amor y la intimidad es inherentemente deshumanizante, independientemente de lo bueno que resulte ser el producto(3).

La postura conservadora de Kass plantea que con el uso de las tecnologías reproductivas, además de jugar a ser Dios, posiblemente nos deshumanicemos. Esta afirmación puede resultar exagerada, ya que no son únicamente las tecnologías reproductivas las que persiguen utilizar el nuevo conocimiento biomédico para mejorar la salud humana o mejorar al humano. Además, tenemos conciencia de las imperfecciones de los avances tecno- 
científicos, mientras que Dios buscaría al menos la perfección. Sin embargo, podemos coincidir con Kass en cuanto a que tenemos la responsabilidad bioética de atender al problema del uso de las tecnologías reproductivas desde una mirada más equilibrada, que no conduzca a la sustitución de la relación sexual.

Hans Jonas, en Técnica, Medicina y Ética, argumenta que la falta de fertilidad, imposibilidad del embarazo o de la reproducción, no son en verdad enfermedades; sin embargo, pueden convertirse en una desgracia, tanto privada como pública, y el médico se hace de algún modo corresponsable de tales desgracias, las cuales podrían evitarse. Jonas afirma que existe responsabilidad, incluso por la vida humana germinal, y que, para suspenderla, la responsabilidad contrapuesta debe tener un peso moral importante. Además, para él, la ciencia y el arte médico tienen una responsabilidad especial, porque solo pueden idear y aplicar los métodos humanos éticamente defendibles(4). La FIV y la gestación subrogada nos sitúan frente a nuevos dilemas bioéticos que bien podrían ser tratados desde la previsión y la responsabilidad, en el sentido de que el hombre, al vivir entre hombres, es siempre responsable de alguien más(4:172), aunque ese alguien no haya nacido.

En contraparte, la postura liberal se enfoca en la decisión de los individuos, en las virtudes de la libertad reproductiva, en el proyecto individual o de pareja. Esta postura defiende el acceso justo a las nuevas formas de reproducción, sugiere que deben estar abiertas a todos y que debería eliminarse cualquier restricción en su acceso, como, por ejemplo, limitarlo a parejas legalmente casadas. Dicha posición sostiene también que la gente que ejercita su autonomía y derecho a la libertad de procreación utilizando formas alternativas, no debería estar en una posición peor o en desventaja respecto de los matrimonios tradicionales(5).

Para esta postura, es más importante obtener el permiso, el consentimiento de las partes involucradas en la FIV y gestación subrogada, que la integridad de los embriones, o si la gestación subrogada se hace de forma altruista o comercial. Apoya todas las posibilidades de formatos de pareja, ya que apela al libre ejercicio de los personas para formar una familia y el derecho reproductivo de mujeres y hombres, sin importar cuál sea su preferencia sexual.

John Harris, quizás el filósofo más liberal dentro de la bioética, afirma en "The Future of Human Reproduction: Ethics, Choice, and Regulation" que es necesaria la tolerancia a la innovación y el respeto de la decisión individual en el uso de las tecnologías de reproducción(6). Argumenta que los que ejercen su libertad reproductiva no necesitan demostrar el bien que puede ocasionar, porque la reproducción es buena de por sí. Más bien, quienes tienen que demostrar sus opiniones son aquellos que cercenan la libertad reproductiva, afirmando que es un procedimiento impopular o indeseable, así como seriamente nocivo para la sociedad en el presente y en el futuro. En realidad, afirma, los conservadores son unos tiranos imaginativos que siempre ponen en riesgo la libre elección de los individuos, imaginando daños que pueden ser posibles pero improbables(7).

La postura liberal funda la aceptación a la FIV y a la gestación subrogada en el principio de autonomía, porque este asegura el respeto del individuo y está vinculado directamente a sus preferencias y derechos individuales. Por su parte Charlesworth, bioeticista liberal, en La Bioética en una Sociedad Liberal(5:76-69) aplica este principio a la reproducción desde la misma mirada; su idea es la de un proyecto individual o de pareja, sin tomar en cuenta algunos límites que puede haber en la autonomía, por lo cual cabe cuestionar en este aspecto a esta postura, pues sí hay límites a la autonomía reproductiva.

\section{Límites de la autonomía reproductiva}

La autonomía reproductiva requiere como mínimo ser racional, es decir, no puede ser arbitraria, lo cual conlleva un proceso de reflexión que, en bioética, implica un diálogo. Por tanto, la autonomía no es individual y momentánea, sino que involucra a la comunidad y exige pensar a largo plazo.

La autonomía racional reproductiva, para tener en verdad un carácter bioético, no debe ser entendida como una mera expresión de deseo; por el contrario, requiere de un proceso exhaustivo de información y de un diálogo y análisis reflexivo 
con el médico, e incluso de educación sexual y reproductiva.

Para establecer límites frente al principio de autonomía y con ello tratar de proteger a la mujer y a los posibles nacidos por FIV, primero habría que modular la autonomía y ponerle condiciones racionales; no es que se limite la autonomía en sí sino la irracional.

La autonomía racional es ética y permitiría a la mujer ejercer su libertad reproductiva con plena conciencia de sus actos, salvaguardando su salud física, emocional, económica y social y la de los posibles nacidos por técnicas de reproducción asistida. Esta forma de actuar podría extenderse a cualquier sujeto posible que participe en los procesos de reproducción artificial: donadores de gametos, mujer gestante, madre genética, incluso a la pareja, aunque aporte o no material genético.

Por el contrario, cuando se ejerce la autonomía de manera irracional, no hay plena conciencia del acto, y no es extraño que la mujer sometida a la FIV tome decisiones no muy racionales, debido a que hay una alteración de su estado emocional durante el tratamiento. La mujer es sometida a dosis de hormonas ${ }^{4} \mathrm{y}$ a posibles y numerosos fracasos durante todo el proceso reproductivo. La FIV provoca ansiedad en muchos de los casos; además, el carácter de imprevisibilidad del resultado del tratamiento puede causar depresión(8). Por ende, la evaluación emocional de la mujer que participa en la FIV o en la gestación subrogada es tan importante y necesaria como su evaluación física.

Aunque la reflexión acerca de los límites de la autonomía reproductiva es exclusivamente de las personas que desean procrear, no está de más que tanto las clínicas y hospitales especializados en tratamientos de reproducción de servicio privado como las instituciones públicas dedicadas a la atención de la infertilidad brinden al paciente la información sobre las consecuencias del embarazo múltiple y otras complicaciones, incluyendo las emocionales, y restrinjan la atención a perso-

${ }^{4} \mathrm{La}$ inducción de la ovulación se efectúa administrando $5.000 \mathrm{UI}$ de hormona gonadotropina coriónica (HCG), cuando los folículos alcanzan un diámetro de $21 \mathrm{~mm}$ y, en todos los casos, la fase lútea deberá ser suplementada con progesterona parenteral o preferentemente micronizada: vías oral o vaginal. nas que no cumplan con los mínimos para llevar a cabo el tratamiento, como salud física, emocional, social, e incluso económica. Y, en caso de solicitar los servicios de una mujer gestante, garantizar que se comprometa a cumplir con el compromiso de entregar al hijo a la pareja o persona sola con la que hizo el acuerdo.

\section{Responsabilidades bioéticas en la reproduc- ción asistida}

El especialista en reproducción asistida no solo es responsable de sí, de la conciencia con que actúa y del modo de llevar a cabo la reproducción, sino también de las condiciones de nacimiento de los que vendrán al mundo a través de estas tecnologías. La responsabilidad ética es la capacidad de respuesta consciente de nuestros actos ante los demás, por tanto, se afirma en la intersubjetividad humana.

Entre las responsabilidades bioéticas en la reproducción asistida están: defender la dignidad ${ }^{5}$ de las personas vulnerables, cuidar la seguridad de los tratamientos de fertilidad, establecer con claridad los lineamientos para su aplicación y contar con la infraestructura adecuada para el almacenamiento de gametos y embriones. Asimismo, contar con guías clínicas y protocolos para el manejo del paciente de la FIV, evaluar psicológicamente a la pareja o persona individual que solicita tal tratamiento y dar seguimiento durante la gestación y después del nacimiento.

Por otro lado, el Estado tiene la responsabilidad de: promulgar leyes que protejan a los usuarios y nacidos con estas tecnologías, brindar educación sexual, contar con programas sociales para apoyar a las mujeres en pobreza extrema, incluso establecer un organismo que gestione el almacenamiento de células germinales y embriones sobrantes de la reproducción asistida. Esto no quiere decir que, al existir todo esto, el Estado controle la libertad reproductiva; por el contrario, esto solo garantizaría que se protejan derechos y libertades, se asegure el acceso a la salud, y principalmente se vigile la seguridad de los tratamientos de reproducción en los sistemas público y privado.

\footnotetext{
${ }^{5}$ Entiéndase por dignidad la forma de trato, consideración y respeto que hemos de tener para los otros pero también para nosotros mismos.
} 
La responsabilidad ética de actuar conforme a los principios que hemos señalado se extiende hacia las células germinales y embriones, cuya protección no solamente debería quedar en manos de los diferentes centros, clínicas, hospitales e institutos, a pesar de que cuenten con la autorización para el manejo de tejidos humanos y células germinales. Un órgano regulador autónomo debería encargarse del control y almacenamiento de dicho material biológico, para asegurar que su manejo sea adecuado y ético. Dicho organismo podría ofrecer un mejor control y asegurar el resguardo de la información confidencial (genética y personal) de los donantes de embriones y células germinales, y podría también fungir como mediador de la donación, en lugar de hacerse de manera privada y posiblemente en la oscuridad. También podría encargarse de establecer parámetros para la destrucción de embriones y su distribución a los centros de investigación, si fueron destinados a este fin.

Por último, considerar que la responsabilidad ética en los tratamientos de reproducción asistida no radica exclusivamente en la ética del presente, sino también en la del futuro, como lo plantea Peter Kemp en "La Mundialización de la Ética", que contempla la proyección del mundo actual y de los seres humanos que aún no nacen(9). Desde una postura éticamente responsable, tendríamos que reflexionar sobre el problema de sobrepoblación que enfrenta el planeta, que sostiene hoy a más de 7.000.000.000 de personas, por lo cual cabe reconsiderar tratamientos de reproducción asistida de manera indiscriminada.

\section{Hacia la figura de un consejero reproductivo}

Las clínicas y hospitales especializados en reproducción debieran contar con un consejero reproductivo, el cual sería una figura similar a la del consejero genético, que ya existe y que ha demostrado ser muy útil y necesario. El consejero reproductivo debiera ser un bioeticista con experiencia en la discusión bioética y tener un comportamiento ético en el ejercicio de su profesión; podría ayudar en la toma de decisiones a las personas que estén en tratamientos de fertilidad; asimismo, ser parte del equipo de salud del hospital o clínica que brinde tratamientos de fertilidad, ya sea de tiempo completo o bien como consejero externo; conocer en profundidad las técnicas de reproducción utilizadas en el hospital o clínica al que apoye con su servicio, y tener conocimientos de genética, psicología, resolución de conflictos y toma de decisiones.

Antes de iniciar el tratamiento, la pareja se presenta con una serie de expectativas frente al médico, con el deseo de procrear un hijo, ya con el mismo genoma de esta o con uno proveniente de la donación de células germinales o de embriones. En algunos casos se necesitará de una mujer gestante que lleve a cabo el embarazo. El especialista en bioética tendría la obligación de comunicar toda la información relevante para la toma de decisiones; principalmente los riesgos del tratamiento; el índice de éxito de la FIV; las implicaciones de salud para la mujer (sea la madre genética o la mujer donante) durante la estimulación ovárica y la extracción de óvulos; el número de embriones que se requiere obtener y el número de embriones que se implantarán por cada intento; las complicaciones de salud en los posibles nacidos, en caso de embarazo múltiple, o la posibilidad de solicitar una mujer gestante como última opción en caso de fracasos repetidos. Dicho de otra forma, el consejero reproductivo puede explicar a profundidad los detalles del tratamiento (técnicos y éticos) para que la pareja o persona individual decida de acuerdo con el caso en que se encuentre.

El consejero reproductivo podría ayudar a la pareja o a los individuos a tomar decisiones con una conciencia ética; ayudar a conocer las mejores opciones, dependiendo de su caso; cuántos intentos desean realizar; qué desean hacer con los embriones sobrantes; qué hacer en caso de frustración e, incluso, podría canalizar a las personas interesadas a terapia psicológica, en caso de solicitarlo.

El propósito de dicho consejero sería enlazar al paciente con el especialista y no dejar vacíos antes y durante el proceso de fertilización. Incluso, orientarlos (en caso de estar fuera del país de origen) sobre la legislación nacional y extranjera y las limitaciones respecto del estatus de los menores al nacer, y respecto de su nacionalidad y otros problemas que surgen con el uso no ético de células germinales de donantes y de mujeres gestantes.

El consejero reproductivo puede también ayudar 
a la pareja a elegir a la mujer gestante que cumpla con mínimos éticos, como: ser mayor de edad, saludable, en una situación económica estable (no en pobreza o extrema pobreza), sin deseo de superarse económicamente a través de la gestación subrogada; tener un nivel de educación medio superior, como mínimo, y así entender la complejidad del tratamiento; ser psicológicamente capaz de tomar decisiones libres de influencias externas; ser capaz de comprender ampliamente las cuestiones técnicas y éticas que implica la gestación subrogada y, sobre todo, que acepte el firme compromiso de entregar al recién nacido. Además, dicha mujer no debería participar más de dos veces en un proceso de gestación, no solamente por cuestiones de salud, sino para evitar la instrumentalización de su cuerpo.

Es indispensable que los solicitantes se informen y reflexionen, antes de comprometerse con el tratamiento, sobre el marco legal que los ampara y los compromete, sobre las responsabilidades que adquieren y sus consecuencias, sobre los diferentes sentimientos que pueden experimentar, así como sobre los obstáculos y riesgos. Esto puede lograrse mediante la figura de este consejero.

\section{Conclusiones}

Este artículo ha intentado exponer los pro y los contra de la FIV y gestación subrogada, ya que ambas han cambiado el paradigma de la reproducción. Fueron planteados algunos dilemas que pueden surgir al respecto, los cuales podrían ser analizados con la ayuda del consejero reproductivo. También se planteó el diálogo entre las posturas predominantes en el discurso bioético, con la finalidad de equilibrar la mirada ante dichos fenómenos. Los límites sugeridos en la autonomía reproductiva se proponen como mecanismo para proteger principalmente a la mujer y a los nacidos por medio de tecnologías reproductivas o gestación subrogada. En cuanto a la responsabilidad bioética, no solamente debemos procurar la seguridad de los tratamientos, sino también la regulación por parte del Estado, sin que esto limite la libertad de los que acuden a la reproducción asistida. Por último, la propuesta de la figura del consejero reproductivo invita a las clínicas y los hospitales especializados en reproducción a realizar un trabajo mucho más ético e intenta proteger a todas las partes involucradas. Implementar esta consejería puede ayudar a brindar una mejor atención en instituciones públicas o privadas.

\section{Referencias}

1. Charlesworth M. La bioética en una sociedad liberal. Gran Bretaña: Cambridge University Press; 1996: 76-79.

2. Sagols L. Interfaz bioética. México: Fontamara; 2006: 115-116.

3. Kass L. La sabiduría de la repugnancia. En: Luna F, Rivera E, (comp.) Los desafíos éticos de la genética humana. México: UNAM, FCE; 2005: 183-198.

4. Jonas H. Técnica, medicina y ética. La práctica del principio de responsabilidad. España: Paidós Básica; 1997: 102-104.

5. Charlesworth M. La bioética en una sociedad liberal. Gran Bretaña: Cambridge University Press; 1996.

6. Harris J, Holm S. The future of human reproduction: ethics, choice, and regulation. New York: Oxford University Press; 1998: 5-37.

7. Harris J. Enhancing evolution. The ethical case for the making better people. New Jersey: Princeton University Press; 2007: 74.

8. Verhaak C, Smeenk J, Evers A, Kremer J, Kraaimaat F, Braat D. Women's emotional adjustment to IVF: a systematic review of 25 years of research. Human Reproduction Update 2007; 13(1): 27-36.

9. Kemp P. La mundialización de la ética. México: Fontamara; 2007: 24. 